\title{
Study of the Best Orientation for Parabolic Concentrator Kind of PolyTrough
}

\author{
Sharif Mohamed Alamen ${ }^{1}$
}

\begin{abstract}
In this study, the best guide for Compound Parabolic Concentrator kind of (PolyTrough) has been evaluated by controlling the angle of incidence of beam radiation. The PolyTrough track the radiation of the sun by moving in prescribed ways to minimize the angle of incidence of the beam radiation on their surfaces and thus maximize the incident beam radiation. Tracking systems are classified according to their motions and rotations into five systems and they are: single axis, which is usually horizontal east-west and moving once (system I), continuously ( System II ), horizontal north-south ( system III,IV) moving continuously and parallel to the earth's axis respectively, or it can be about two axes (system V). We estimated the amount of direct solar radiation in each system controller and then the comparison between the different control methods has been estimated. The results of the study have showed that the system $\mathrm{V}$ collects the largest amount of direct solar radiation, but the cost of this system is very expensive due to the presence of two types of collector's motion. While the fourth (IV) system, is distinguished from the rest of the systems, because it collects a large amount of solar radiation in the winter and decreases in the summer, where this system depends on the angle of the latitude of the site. The control system of the third kind (System III), which is less expensive than the type $\mathrm{V}$ and as it moves around one axis only, it collects the amount of solar energy approaching the system $\mathrm{V}$ especially in summer. The systems I and II collected values are close to each other, and although that these two systems collect a small amount of the solar radiation during the day comparing to the rest of the systems, this small amount of radiation is considered to be acceptable in the some studied areas.
\end{abstract}

Keywords: PolyTrough, angle of incidence, direct solar radiation

\section{Introduction}

The geometric relationships between a plane of any particular orientation relative to the earth at any time (whether that plane is fixed or moving relative to the earth) and the incoming beam solar radiation, that is, the position of the sun relative to that plane, can be described in terms of several angles. Some solar collectors track the sun by moving in prescribed ways to minimize the angle of incidence of beam radiation on their surfaces and thus maximize the incident beam radiation. The angles of incidence and the surface azimuth angles are needed for these collectors. Relationships in this section will be useful in radiation calculations for these moving surface. Tracking systems are classified by their motions. Rotation can be about a single axis (which could have any 
orientation but which in practice is usually horizontal east-west, horizontal north-south, vertical, or parallel to the earth's axis) or it can be about two axis[1].

\section{Estimate the amount of direct solar radiation}

Statistical studies of the time distribution of total radiation on horizontal surfaces through the day, using monthly average data for a number of stations, have let to generalized charts of $\mathrm{r}_{\mathrm{T}}$, the ratio of hourly total to daily radiation, as a function of day length and the hour in question:

$r_{T}=\frac{I_{T}}{H_{m}}=\frac{\pi}{24}(c+d \cos \omega) \frac{\cos \omega-\cos \omega_{s}}{\sin \omega_{s}-\frac{\pi \omega_{S}}{180} \cos \omega_{s}}$

Where: $H_{m}$ is the monthly average daily global radiation on a horizontal surface $\left(\mathrm{MJ} \cdot \mathrm{m}^{-}\right.$ $2 \cdot$ day $^{-1}$ ), represented by the expression $H_{m}=H_{o} K_{T}$, where $K_{T}$ is the daily clearness Index me by given by[1],[9]:

$K_{T}=a+b \frac{n}{N}$

Where $n$ is the monthly average daily number of hours of bright sunshine, $N$ is the monthly average daily maximum number of hours of possible sunshine (or day length), $a$ and $b$ are regression constants[6].

$H o$ is the extraterrestrial solar radiation on a horizontal surface [1].

The coefficient $c$ and $d$ are given by:

$c=0.409+0.5016 \sin \left(\omega_{s}-60\right)$

$d=0.6609+0.4767 \sin \left(\omega_{s}-60\right)$

In these equations $\omega$ is the hour angle in degrees for the time in question, and $\omega_{\mathbf{s}}$ is the sunset hour angle[5].

We can be used to estimate hourly average of diffuse radiation, If the average daily total radiation is known:

$r_{d}=\frac{I_{d}}{H_{d}}=\frac{\pi}{24} \frac{\cos \omega-\cos \omega_{s}}{\sin \omega_{s}-\frac{\pi \omega_{s}}{180} \cos \omega_{s}}$

We know that hourly beam radiation is $I_{b}=I_{T}-I_{d}$ and the direct solar radiation coming from the sun disk is $I_{b n}=I_{b} / \cos \theta_{z}$; thus we can estimate the amount of direct solar radiation falling on the surface of the solar collector (PolyTrough) in this equation[7]:

$D N I=I_{b n} \cos \theta$

The angle of incident $\theta$ is needed for these collector. The following sets of equations are for surfaces that rotate on axes that are parallel to the surface. For a PolyTrough rotated 
about a horizontal east-west axis with a single daily adjustment so that the beam radiation is normal to the surface at noon each day,

$\cos \theta=\sin ^{2} \delta+\cos ^{2} \delta \cos \omega$

For a PolyTrough rotated about a horizontal east-west axis with continuous adjustment to minimize the angle of incidence,

$\cos \theta=\left(1-\cos ^{2} \delta \sin ^{2} \omega\right)^{0.5}$

For a PolyTrough rotated about a horizontal north-south axis with continuous adjustment to minimize the angle of incidence,

$\cos \theta=\left(\cos ^{2} \theta_{z}+\cos ^{2} \delta \sin ^{2} \omega\right)^{0.5}$

For a Poly'Trough rotated about a horizontal north-south axis parallel to the earth's axis with continuous adjustment to minimize the angle of incidence,

$\cos \theta=\cos \delta$

For a Poly'Trough that is continuously tracking about tow axes to minimize the angle of incidence, $\quad \cos \theta=1$

\section{Results and discussion}

A computer program was written to estimate the amount of direct solar radiation $(D N I)$. The program was run under the following inputs:

- Was chosen five different areas latitudes and regression constants as shown in Table 1.

Table 1: The different areas latitudes and regression constants

\begin{tabular}{|l|l|l|l|l|}
\hline & City & Latitude & $\mathbf{a}$ & b \\
\hline 1 & Karonga, Malawi & 9.57 & 0.31 & 0.44 \\
\hline 2 & Sanaa, Yamen & 15.52 & 0.347 & 0.364 \\
\hline 3 & Sebha, Libya & 27.02 & 0.3 & 0.43 \\
\hline 4 & Sirte, Libya & 31.12 & 0.367 & 0.342 \\
\hline 5 & Nice, France & 43.42 & 0.17 & 0.63 \\
\hline
\end{tabular}

- $\quad$ The monthly average daily number of hours of bright sunshine (hours) for five different regions Latitude;

- $\quad$ The solar constant $\left(I_{s c}=1367 \mathrm{~W} \mathrm{~m}^{-2}\right)$;

- $\quad$ The day for each month of the year.

The following curves $(1,2,3,4,5)$ show the amount of direct solar radiation (DNI) for five different latitude, where was estimated direct solar radiation in five different systems. The system $\mathrm{V}$ collects the largest amount of direct solar radiation, but the cost of this system is very expensive due to the presence of two types of collector's motion. While the fourth (IV) system, is distinguished from the rest of the systems, because it collects a 
large amount of solar radiation in the winter and decreases in the summer, where this system depends on the angle of the latitude of the site. The control system of the third kind (System III), which is less expensive than the type $\mathrm{V}$ and as it moves around one axis only, it collects the amount of solar energy approaching the system $\mathrm{V}$ especially in summer. The systems I and II collected values are close to each other, and although that these two systems collect a small amount of the solar radiation during the day comparing to the rest of the systems, this small amount of radiation is considered to be acceptable in the some studied areas, curves $(3,4)$ (Sebha, Sirte Libya).

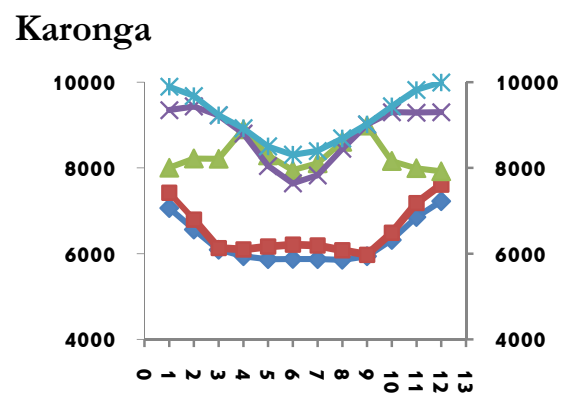

(1)

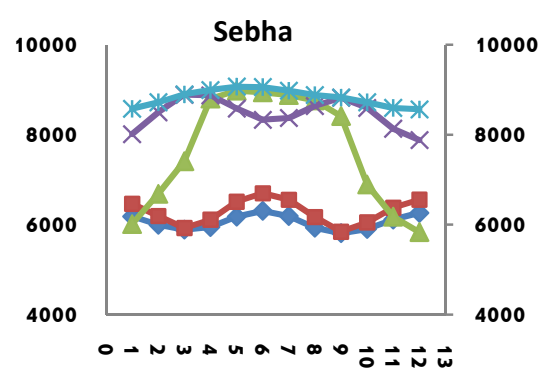

(3)

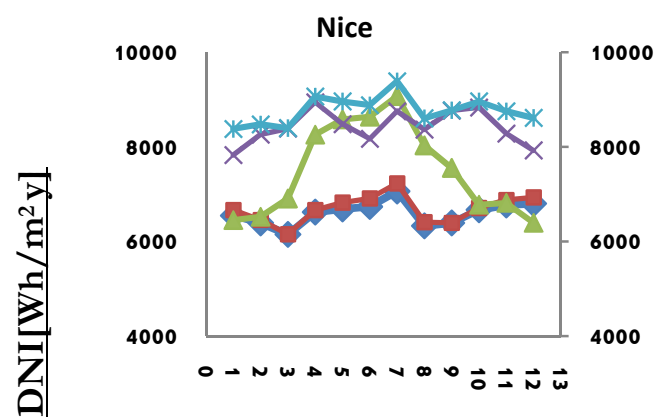

(5)

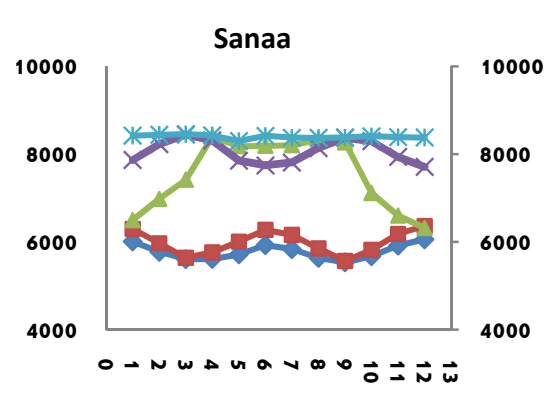

(2)

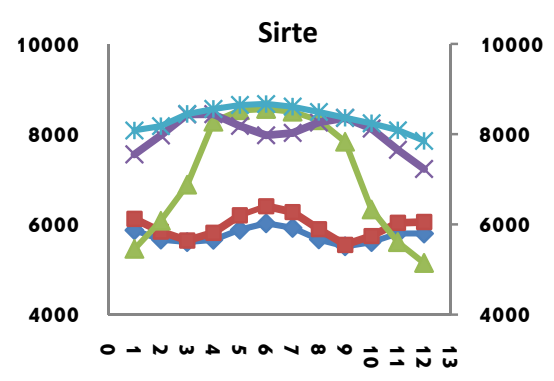

(4)

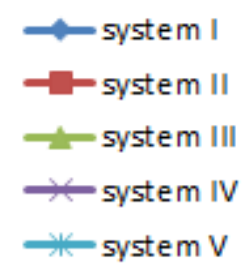

$\underline{\text { Month }}$ 


\section{Conclusion}

It is noted that the control system $V$ gave the best amount of direct solar radiation throughout the day, but the cost of this system is very expensive due to the presence of two types of collector's motion; followed by a fourth system, then the third. Second and first system collected small amount of the solar radiation during the day comparing to the rest of the systems, this small amount of radiation is considered to be acceptable in the some studied areas (e.g. Libya).

\section{References}

[1] Duffie Beckman "Solar engineering of thermal processes", second edition, 1991

[2] Empirical Model for the Estimation of Global Solar Radiation in Makurdi, Nigeria, Volume 12 Issue 1 Version 1.0 January 2012.

[3] Isikwue, B.C. Audu, M.O. and Utah, E.U. "Empirical Model for Estimating Diffuse Fraction of Solar Radiation over Makurdi, Nigeria". International Journal of Science and Advanced Technology, vol. 2, no. 2, pp 5-10, 2012b.

[4] A. Angstrom, "Solar and Terrestrial Radiation", Quarterly Journal of the Royal Meteorological Society, Vol. 50, No. 210, 1924, pp. 121-125.

[5] Turton, S.M. "The Relationship between Total Irradiation and Sunshine

Duration in the Humid Tropics". Solar Energy. Vol. 38: pp 353-354, 1986.

[6] Akinoglu B.G., Ecevit A. 1990. Construction of a quadratic model using modified Angstrom coefficients to estimate global solar radiation, Solar Energy, 45, 85-92.

[7] Samuel T.D.M.A. 1991. Estimation of global radiation for Sri Lanka, Solar Energy, 47, 333-337.

[8] Government of Malawi, "Malawi State of Environment and Outlook: Environment for Sustainable Economic Growth," Malawi Government , 2010.

[9] Determining Angstrom Constants for Estimating Solar Radiation in Malawi, Griffin Salima, Geoffrey M. S. Chavula, International Journal of Geosciences, 2012, 3, 391-397. 
